\title{
Características de carcaça de galinha naturalizada Peloco comparada a linhagens de frango caipira
}

\author{
Eva Clícia de Jesus Almeida(1), Paulo Luiz Souza Carneiro(1), Amauri Arias Wenceslau(2), \\ Ronaldo Vasconcelos Farias Filho ${ }^{(3)}$ e Carlos Henrique Mendes Malhado(1)
}

\begin{abstract}
(1) Universidade Estadual do Sudoeste da Bahia, Departamento de Ciências Biológicas, Rua José Moreira Sobrinho, s/no, Jequiezinho, CEP 45206-190 Jequié, BA, Brasil. E-mail: evaclicia@gmail.com, plscarneiro@gmail.com, carlosmalhado@gmail.com (2)Universidade Estadual de Santa Cruz, Departamento de Ciências Agrárias e Ambientais, Rodovia Ilhéus-Itabuna, Km 16, Campus Universitário, CEP 45662-900 Ilhéus, BA, Brasil. E-mail: amauriwenceslau@yahoo.com.br (3)Universidade Estadual do Sudoeste da Bahia, Faculdade de Zootecnia, Rodovia BR-415 Km 03, s/no, CEP 45700-000 Itapetinga, BA, Brasil. E-mail: rvff50@gmail.com
\end{abstract}

Resumo - O objetivo deste trabalho foi avaliar a diversidade fenotípica de uma população de galinhas Peloco e compará-la a três linhagens comerciais de frango de corte do tipo caipira. Avaliações foram feitas quanto a características de: peso de carcaça, peito, coxa e sobrecoxa; altura, largura e comprimento do peito; e comprimento da coxa e da sobrecoxa. Os dados foram submetidos às análises de variáveis canônicas e à análise discriminante independente do tamanho (ADIT). Os resíduos da ADIT foram utilizados na análise de agrupamento pelos métodos de Tocher e UPGMA. As duas primeiras variáveis canônicas foram suficientes para explicar $94,66 \%$ da variação total entre os fenótipos. O peso da carcaça teve a maior contribuição para a variação (59,91\%) entre os grupos de aves, seguido de peso da coxa $(12,63 \%)$ e largura do peito $(11,75 \%)$. Ambos os métodos de agrupamento resultaram na formação de dois grupos: um de linhagens comerciais e outro da raça Peloco. O isolamento da Peloco é consequência da ausência de seleção para as características estudadas. Além disso, a presença de variabilidade dentro da raça Peloco mostra a existência de animais com maior potencial quanto ao peso de carcaça, o que possibilita o melhoramento da raça.

Termos para indexação: diversidade fenotípica, análise multivariada, recursos genéticos.

\section{Carcass characteristics of Peloco naturalized chicken compared to free-range broiler lineages}

\begin{abstract}
The objective of this work was to evaluate the phenotypic diversity of a Peloco chicken population and to compare it with three free-range broiler commercial lineages. Evaluations were done for the following characteristics: the weights of carcass, breast, drumstick, and thigh; the height, width, and length of breast; and the lengths of drumstick and thigh. Data were submitted to canonic variable analyses and to size-independent discriminant analysis (ADIT). The ADIT residues were used in Tocher's grouping analysis and in UPGMA method. The first two canonic variables were sufficient to explain $94.66 \%$ of the total variation among phenotypes. Carcass weight had the greatest contribution for the variation $(59.91 \%)$ among the bird groups, followed by drumstick weight (12.63\%) and breast width (11.75\%). Both grouping methods resulted in the formation of two groups, one of the commercial lineages, and other of the Peloco breed. Peloco isolation is a consequence of the selection absence for the studied characteristics. In addition, the presence of variability in Peloco breed shows the existence of animals with a greater potential for carcass weight, which makes it possible the improvement of this breed.
\end{abstract}

Index terms: phenotypic diversity, multivariate analysis, genetic resources.

\section{Introdução}

As galinhas naturalizadas brasileiras surgiram de cruzamentos aleatórios entre as raças trazidas pelos portugueses na época da colonização (Egito et al., 2002). Embora muitas delas tenham desaparecido, algumas ainda são encontradas nas mais diversas regiões do país. As aves naturalizadas são úteis para agricultores com poucos recursos, pois podem ter boa produtividade, sem a necessidade de altos investimentos, e requerem menor controle sanitário (Yakubu \& Ugbo, 2011), o que contribui para a qualidade de vida das famílias rurais de baixa renda (Kaya \& Yildiz, 2008) e para sua segurança alimentar (Hailemariam et al., 2010).

No Estado da Bahia há grupos de galinhas naturalizadas, sobre cuja origem ou local de origem pouco ou nada se sabe. A galinha da raça Peloco está 
presente em fazendas e criações caseiras na Chapada Diamantina e nas regiões sudoeste, sul e extremo sul do Estado. As aves são criadas exclusivamente em sistema extensivo, com pouco investimento tecnológico, ao qual são bem adaptadas, e caracterizam-se pela ausência de penas, durante longo período da fase de crescimento, e coloração escura da carne. Além do pouco investimento tecnológico empregado em sua criação, o seu potencial para a produção de carne e de ovos é ainda desconhecido.

Os estudos sobre as características da carcaça em avicultura são cruciais, pois estas são critérios de seleção em programas de melhoramento. Para as raças naturalizadas estes estudos contribuem ainda para a caracterização zootécnica das aves. Algumas abordagens sobre desempenho de crescimento e carcaça das raças nativas de galinhas são relatadas na literatura (Zanetti et al., 2010; Kgwatalala et al., 2012), inclusive também a comparação entre genótipos nativos e comerciais (Franco et al., 2012).

As galinhas naturalizadas são uma alternativa para a sustentabilidade do pequeno agricultor, e suas características podem ser aproveitadas na produção avícola. Apesar de não haver referências na literatura sobre a potencialidade da raça Peloco, nas condições em que são criadas, sem o mínimo controle zootécnico, mostram-se aparentemente mais resistentes a parasitas e adaptadas ao clima quente do que os genótipos comerciais; portanto, estudos sobre esta raça são necessários.

O objetivo deste trabalho foi avaliar a diversidade fenotípica de uma população de galinhas Peloco e compará-la a três linhagens comerciais de frango de corte do tipo caipira.

\section{Material e Métodos}

O experimento foi conduzido no Setor de Avicultura da Universidade Estadual do Sudoeste da Bahia (Uesb), no Município de Itapetinga, BA, de fevereiro a julho de 2012. Foram utilizados dados de carcaça de 157 aves - machos e fêmeas -, de quatro grupos genéticos, das quais 40 aves comerciais de frango de corte do tipo caipira, para cada linhagem (L1, L2 e L3), provenientes da aquisição de pintainhos de um dia de uma granja especializada. A L1 foi composta por aves Master Griss, selecionadas para atingir 2,20 kg dos 56 aos 68 dias de idade, classificadas como aves de crescimento rápido. AL2 foi composta por aves Redbro, selecionadas para atingir $2,20 \mathrm{~kg}$ dos 70 aos 80 dias de idade, classificadas como aves de crescimento intermediário. A L3 foi composta por aves Acoblack, selecionadas para atingir 2,20 kg dos 90 aos 100 dias de idade, classificadas como aves de crescimento lento. Avaliaram-se 37 aves da raça de galinha nativa Peloco, oriundas de um plantel de 48 fêmeas e seis machos, do Setor de Avicultura da Uesb.

Todas as aves foram criadas em sistema de confinamento, com fornecimento de água, forragem e ração ad libitum. As rações utilizadas na fase inicial e de crescimento foram formuladas à base de milho e farelo de soja, e sua composição seguiu as exigências nutricionais de cada fase (Rostagno et al., 2011).

A idade de abate das galinhas de cada linhagem foi fixada de acordo com a recomendação do fornecedor, para que não houvesse modificações nas características de carcaça, em razão da respectiva biologia de crescimento. A L1 foi abatida aos 65 dias de idade; a L2 aos 80 dias; e a L3 aos 100 dias. As aves Peloco foram abatidas aos 150 dias, idade utilizada nas comunidades onde são criadas. Todas as aves foram abatidas por meio de ruptura da veia jugular e evisceradas após sangria e depenação.

Foram mensuradas as seguintes características: os pesos de carcaça (PCA), peito (PPT), coxa (PCX) e sobrecoxa (PSC); o comprimento (CPT), a altura (APT) e a largura do peito (LPT); e os comprimentos da coxa (CCX) e da sobrecoxa (CSC). As medidas de peso e de carcaça foram feitas com balança (precisão $0,005 \mathrm{~kg}$ ) e paquímetro digital (precisão $0,5 \mathrm{~mm}$ ), respectivamente.

Os dados foram submetidos à análise de variância, para avaliar os efeitos de sexo e grupo genético. As médias foram comparadas pelo teste de Tukey a $5 \%$ de probabilidade. Utilizou-se o modelo estatístico,

$$
\mathrm{Y}_{\mathrm{itr}}=\mu_{\mathrm{t}}+\mathrm{S}_{\mathrm{it}}+\mathrm{G}_{\mathrm{it}}+\left(\mathrm{S}_{\mathrm{it}} \times \mathrm{G}_{\mathrm{it}}\right)+\varepsilon_{\mathrm{itr}},
$$

em que: $Y_{\text {itr }}$ é o valor da característica t, no grupo genético $i$, no animal $r$; $\mu_{\mathrm{t}}$ é a média da característica t; $S_{i t}$ é o efeito do sexo i, na característica t; $G_{i t}$ é o efeito do grupo genético i, na característica t; $\left(\mathrm{S}_{\mathrm{it}} \times \mathrm{G}_{\mathrm{it}}\right)$ é o efeito da interação do sexo i e grupo genético i na característica t; e $\varepsilon_{\text {itr }}$ é o efeito do erro aleatório atribuído à observação $Y_{\text {itr }}$.

As características mensuradas foram submetidas à análise multivariada prévia, como objetivo deidentificar 
variáveis redundantes para a variação global $\left(\mathrm{S}_{\mathrm{j}}\right)$, por meio da avaliação da contribuição das variáveis para a variação entre os grupos genéticos. Após a primeira avaliação, foram descartadas três variáveis (CPT, APT e CCX), tendo-se adotado a importância relativa das características para a variação total, como critério de descarte, conforme a metodologia de Singh (1981).

O novo conjunto de variáveis, composto por PCA, PPT, PCX, PSC, LPT e CSC, foi submetido à análise discriminante independente do tamanho (ADIT), para a remoção dos efeitos da variação do tamanho na forma (Reis et al., 1990; Peres-Neto, 1995). Após a regressão de cada variável em função do primeiro componente principal, os resíduos foram usados numa análise multivariada.

A diversidade fenotípica entre os grupos foi obtida por meio da análise de variáveis canônicas e análise de agrupamento. Como medidas de dissimilaridade, foram adotadas a distância generalizada $\mathrm{D}^{2}$ de Mahalanobis (Mahalanobis, 1936) e a distância euclidiana média (Cruz \& Carneiro, 2006).

Os gráficos de dispersão da variação intra e interpopulacional foram obtidos com base nos escores das variáveis canônicas, que permitiram a avaliação da divergência fenotípica. Os resíduos gerados pela ADIT foram usados na análise de agrupamento. Utilizou-se o método de otimização de Tocher, tendo-se adotado o critério de que a distância média intragrupo deve ser menor do que a distância média intergrupo.

O método hierárquico UPGMA (Unweighted pair group method with arithmetic mean) foi usado para gerar o dendrograma com os grupos de maior similaridade. Para as análises de variância, ADIT e variáveis canônicas, utilizou-se o programa estatístico SAS versão 9.0 (SAS Institute, Cary, NC, EUA). As análises de agrupamento foram feitas pelo programa Genes (Cruz, 2009), e os gráficos gerados no utilitário Past 2.03 (Hammer et al., 2001).

\section{Resultados e Discussão}

Observou-se que a raça Peloco diferiu dos três grupos genéticos em todas as variáveis, com exceção da APT e CCX com a L1 e L2 e CSC com a L2 (Tabela 1). Verificaram-se diferenças associadas ao sexo, com exceção de PPT e APT, que não diferiram entre machos e fêmeas. Valores médios superiores foram observados para os machos, o que constatou dimorfismo sexual da espécie e, consequentemente, diferenças hormonais quanto ao crescimento entre os sexos.

Houve efeito significativo da interação entre sexo e grupo genético quanto a todas as características biométricas (CPT, APT, LPT, CCX e CSC), tendose observado efeito não significativo quanto às características de peso. No desdobramento da interação, observou-se, quanto à característica CPT, que houve diferença significativa entre machos e fêmeas apenas nas aves do grupo Peloco. Não se constatou efeito significativo de sexo quanto às variáveis APT, LPT e CSC nas linhagens L2 e L3. Quanto à característica CCX, não houve efeito significativo de sexo, a não ser para a linhagem L3.

As galinhas da raça Peloco apresentaram valores médios de peso inferiores aos das três linhagens comerciais. Este resultado foi semelhante ao relatado por Adeleke et al. (2011), em seu estudo do desempenho de crescimento de raças nativas e comerciais na Nigeria. As diferenças de peso corporal entre raças industriais e nativas evidenciam que estas últimas são aves mais leves (Ajayi et al., 2012). Além disso, o uso dessas raças nativas, em cruzamentos com as raças comerciais, pode promover a melhoria no desempenho quanto ao ganho de peso e, ainda, aumentar a rusticidade dos animais selecionados.

Os valores da contribuição relativa das características para a variação global (S.j), em ordem de menor contribuição, foram as seguintes: $\mathrm{CCX}=0,06$, $\mathrm{APT}=1,88$ e CPT $=3,29$ (Tabela 2). Estas três variáveis foram descartadas da análise por serem pouco relevantes para a explicação da variação entre os grupos avaliados. A avaliação da contribuição relativa dos caracteres para a variação total é importante, pois permite identificar variáveis pouco informativas, ou seja, aquelas de menor S.j, que podem ser descartadas com o objetivo de se reduzir a mão de obra e o tempo da coleta de dados em trabalhos futuros e, ainda, melhorar o desempenho das análises (Cruz \& Carneiro, 2006). Após o descarte, não se observou alteração no agrupamento, o que confirmou a redundância destas variáveis (Oliveira et al., 2004).

Atenção especial deve ser direcionada para as características comercialmente importantes e que tiveram maior consideração no estudo da diversidade, que são: PCA, PCX, LPT e PSC. Este resultado devese, em maior parte, à grande variação existente entre as linhagens comerciais, selecionadas desde muito tempo

Pesq. agropec. bras., Brasília, v.48, n.11, p.1517-1523, nov. 2013 DOI: $10.1590 / \mathrm{S} 0100-204 X 2013001100013$ 
quanto ao maior peso corporal e rendimento de carcaça nos programas de melhoramento avícola. A raça Peloco apresentou menor tamanho corporal, como quase todas as raças nativas, provavelmente em razão da seleção natural, que favoreceu a redução do tamanho corporal como forma de garantir sua sobrevivência.

A análise discriminante independente do tamanho (ADIT) mostrou que o primeiro componente principal (CP1) explicou $83,21 \%$ da variação total entre os grupos genéticos; assim, o uso da ADIT mostrou-se eficiente para a correção do efeito do tamanho sobre a forma. Quanto maior o valor da variação explicada pelo primeiro componente principal, maior é a eficiência desta análise (Paiva et al., 2006; Silva et al., 2009).

Todas as variáveis de peso apresentaram correlação significativa e de alta magnitude com os coeficientes do CP1 (Tabela 3). O peso da carcaça foi a variável que apresentou maior correlação $(\mathrm{r}=0,97)$ com o

Tabela 1. Média \pm erro-padrão do peso e de características biométricas da carcaça de machos e fêmeas das linhagens comerciais L1, L2 e L3 e da raça naturalizada Peloco ${ }^{(1)}$.

\begin{tabular}{|c|c|c|c|c|c|}
\hline \multirow[t]{2}{*}{ Sexo } & \multicolumn{3}{|c|}{ Linhagens comerciais } & \multirow[t]{2}{*}{ Peloco } & \multirow[t]{2}{*}{ Média } \\
\hline & L1 & L2 & L3 & & \\
\hline & & & PCA (kg) & & \\
\hline Macho & 1,62 & 2,08 & 2,26 & 1,32 & $1,81 \pm 0,034^{*}$ \\
\hline Fêmea & 1,37 & 1,80 & 2,11 & 0,99 & $1,57 \pm 0,029^{*}$ \\
\hline \multirow[t]{2}{*}{ Média } & $1,49 \pm 0,044 \mathrm{a}$ & $1,93 \pm 0,044 \mathrm{~b}$ & $2,19 \pm 0,043 \mathrm{c}$ & $1,14 \pm 0,045 \mathrm{~d}$ & - \\
\hline & & & PPT (kg) & & \\
\hline Macho & 0,36 & 0,48 & 0,47 & 0,27 & $0,39 \pm 0,009^{\mathrm{ns}}$ \\
\hline Fêmea & 0,31 & 0,44 & 0,46 & 0,21 & $0,37 \pm 0,008^{\mathrm{ns}}$ \\
\hline \multirow[t]{2}{*}{ Média } & $0,34 \pm 0,012 \mathrm{a}$ & $0,46 \pm 0,012 \mathrm{~b}$ & $0,47 \pm 0,012 \mathrm{~b}$ & $0,24 \pm 0,012 \mathrm{c}$ & - \\
\hline & & & PCX $(\mathrm{kg})$ & & \\
\hline Macho & 0,13 & 0,15 & 0,17 & 0,10 & $0,14 \pm 0,003 *$ \\
\hline Fêmea & 0,09 & 0,12 & 0,15 & 0,07 & $0,11 \pm 0,003 *$ \\
\hline \multirow[t]{2}{*}{ Média } & $0,11 \pm 0,004 \mathrm{a}$ & $0,13 \pm 0,004 \mathrm{a}$ & $0,16 \pm 0,004 \mathrm{~b}$ & $0,08 \pm 0,004 \mathrm{c}$ & - \\
\hline & & & PSC (kg) & & \\
\hline Macho & 0,12 & 0,15 & 0,14 & 0,09 & $0,13 \pm 0,003^{*}$ \\
\hline Fêmea & 0,09 & 0,12 & 0,13 & 0,07 & $0,11 \pm 0,002 *$ \\
\hline \multirow[t]{2}{*}{ Média } & $0,11 \pm 0,003 \mathrm{a}$ & $0,14 \pm 0,003 \mathrm{~b}$ & $0,14 \pm 0,003 \mathrm{~b}$ & $0,08 \pm 0,003 \mathrm{c}$ & - \\
\hline & & & $\mathrm{CPT}(\mathrm{mm})$ & & \\
\hline Macho & $172,19 \mathrm{Aa}$ & $177,57 \mathrm{Aa}$ & $174,21 \mathrm{Aa}$ & $168,44 \mathrm{Aa}$ & $172,39 \pm 2,000 *$ \\
\hline Fêmea & $163,24 \mathrm{Aa}$ & $171,54 \mathrm{Aa}$ & $172,58 \mathrm{Aa}$ & $142,78 \mathrm{Bc}$ & $162,98 \pm 1,760 *$ \\
\hline \multirow[t]{2}{*}{ Média } & $167,63 \pm 2,609 a$ & $173,50 \pm 2,595 \mathrm{a}$ & $173,60 \pm 2,573 \mathrm{a}$ & $151,79 \pm 2,683 b$ & - \\
\hline & & & APT (mm) & & \\
\hline Macho & $56,01 \mathrm{Aab}$ & 59,99Aab & $51,93 \mathrm{Ab}$ & $62,86 \mathrm{Aa}$ & $57,26 \pm 1,020^{\mathrm{ns}}$ \\
\hline Fêmea & $48,96 \mathrm{Bac}$ & $57,16 \mathrm{Ab}$ & $51,52 \mathrm{Ac}$ & $54,28 \mathrm{Babc}$ & $54,10 \pm 0,890^{\text {ns }}$ \\
\hline \multirow[t]{2}{*}{ Média } & $53,27 \pm 1,329 \mathrm{ac}$ & $59,62 \pm 1,322 b$ & $52,07 \pm 1,311 \mathrm{a}$ & $57,77 \pm 1,367 \mathrm{bc}$ & - \\
\hline & & & LPT (mm) & & \\
\hline Macho & $133,54 \mathrm{Aac}$ & $84,22 \mathrm{Ab}$ & $81,43 \mathrm{Abc}$ & $64,64 \mathrm{Ad}$ & $76,50 \pm 0,980^{*}$ \\
\hline Fêmea & $126,28 \mathrm{Ba}$ & $82,70 \mathrm{Ab}$ & $79,46 \mathrm{Ab}$ & $57,60 \mathrm{Bc}$ & $72,61 \pm 0,860^{*}$ \\
\hline \multirow[t]{2}{*}{ Média } & $72,50 \pm 1,28 \mathrm{a}$ & $83,88 \pm 1,276 b$ & $81,18 \pm 1,265 \mathrm{~b}$ & $60,66 \pm 1,319 \mathrm{c}$ & - \\
\hline & & & $\mathrm{CCX}(\mathrm{mm})$ & & \\
\hline Macho & $134,60 \mathrm{Aa}$ & $133,54 \mathrm{Aa}$ & $145,73 \mathrm{Ab}$ & $144,71 \mathrm{Ab}$ & $139,68 \pm 1,360^{*}$ \\
\hline Fêmea & $119,07 \mathrm{Ba}$ & $126,28 \mathrm{Bb}$ & $139,24 \mathrm{Ac}$ & $119,91 \mathrm{Bab}$ & $126,41 \pm 1,190^{*}$ \\
\hline \multirow[t]{2}{*}{ Média } & $127,29 \pm 1,769 a$ & $130,96 \pm 1,761 \mathrm{a}$ & $143,33 \pm 1,746 b$ & $130,59 \pm 1,821 \mathrm{a}$ & - \\
\hline & & & $\mathrm{CSC}(\mathrm{mm})$ & & \\
\hline Macho & $89,11 \mathrm{Aa}$ & $95,05 \mathrm{Ab}$ & $101,34 \mathrm{Ac}$ & $102,25 \mathrm{Ac}$ & $97,06 \pm 0,880^{*}$ \\
\hline Fêmea & $78,28 \mathrm{Ba}$ & $92,44 \mathrm{Ab}$ & $98,89 \mathrm{Ac}$ & $85,44 \mathrm{Bc}$ & $89,09 \pm 0,760 *$ \\
\hline Média & $84,27 \pm 1,152 \mathrm{a}$ & $94,68 \pm 1,146 b$ & $100,80 \pm 1,136 \mathrm{c}$ & $92,53 \pm 1,184 \mathrm{~b}$ & - \\
\hline
\end{tabular}

${ }^{(1)}$ Médias seguidas de letras iguais, maiúsculas nas colunas e minúsculas nas linhas, não diferem entre si, pelo teste Tukey, a 5\% de probabilidade. *Significativo a 5\% de probabilidade, pelo teste F. ns Não significativo. PCA, peso da carcaça; PPT, peso do peito; PCX, peso da coxa; PSC, peso da sobrecoxa; CPT, comprimento do peito; APT, altura do peito; LPT, largura do peito; CCX, comprimento da coxa; CSC, comprimento da sobrecoxa. 
coeficiente do $\mathrm{CP} 1$, o que permitiu interpretá-lo como variável relacionada ao tamanho.

As duas primeiras variáveis canônicas ( $\mathrm{VC1}=69,30 \%$ e $\mathrm{VC} 2=25,33 \%$ ) foram suficientes para explicar $94,63 \%$ da variação original (Figura 1). Considerandose que nas primeiras variáveis está contida mais de $80 \%$ da variância total, o estudo da divergência genética entre genótipos, por meio das distâncias geométricas em gráficos de dispersão, torna-se viável (Cruz et al., 2011).

A raça Peloco apresentou maior variabilidade intrapopulacional, enquanto os indivíduos das linhagens comerciais foram mais similares. Isto pode ser explicado pela seleção e, consequentemente, padronização do peso corporal nas linhagens

Tabela 2. Contribuição relativa dos caracteres para a variação global (S.j) quanto à diversidade entre as linhagens comerciais L1, L2 e L3 e a raça naturalizada Peloco.

\begin{tabular}{lrc}
\hline Variável $^{(1)}$ & \multicolumn{1}{c}{$\mathrm{S} . \mathrm{j}^{(2)}$} & $\mathrm{S} . \mathrm{j}(\%)^{(3)}$ \\
\hline PCA & 93,00 & 50,91 \\
PPT & 7,81 & 4,28 \\
PCX & 23,08 & 12,63 \\
PSC & 15,17 & 8,30 \\
CPT $^{*}$ & 6,02 & 3,29 \\
APT $^{*}$ & 3,43 & 1,88 \\
LPT & 21,46 & 11,75 \\
CCX & 0,11 & 0,06 \\
CSC & 12,59 & 6,89 \\
\hline
\end{tabular}

(1)PCA, peso da carcaça; PPT, peso do peito; PCX, peso da coxa; PSC, peso da sobrecoxa; CPT, comprimento do peito; APT, altura do peito; LPT, largura do peito; CCX, comprimento da coxa; CSC, comprimento da sobrecoxa. ${ }^{(2)}$ S.j, contribuição relativa. ${ }^{(3)}$ S.j(\%), valor percentual. *Variáveis de menor contribuição para a variação total (S.j), posteriormente descartadas da análise multivariada.

Tabela 3. Coeficientes do primeiro componente principal (CP1) e valores de correlação simples (r) das variáveis utilizadas na análise discriminante independente do tamanho (ADIT), entre as linhagens comerciais L1, L2 e L3 e a raça naturalizada Peloco.

\begin{tabular}{lcc}
\hline Variável $^{(1)}$ & $\mathrm{CP} 1$ & $\mathrm{r}$ \\
\hline PCA & 0,443 & $0,97^{* *}$ \\
PPT & 0,503 & $0,90^{* *}$ \\
PCX & 0,534 & $0,95^{* *}$ \\
PSC & 0,473 & $0,94^{* *}$ \\
LPT & 0,168 & $0,61^{* *}$ \\
CSC & 0,117 & $0,55^{* *}$ \\
\hline
\end{tabular}

(1) PCA, peso da carcaça; PPT, peso do peito; PCX, peso da coxa; PSC, peso da sobrecoxa; LPT, largura do peito; CSC, comprimento da sobrecoxa. **Significativo a $1 \%$ de probabilidade. comerciais. Em contraste, a raça Peloco não sofreu seleção quanto às características produtivas, apresentando grande variabilidade fenotípica. Acreditase que as galinhas naturalizadas tenham sido formadas por aves de diversos tipos, de tamanho médio a grande como as inglesas, americanas e asiáticas, até as de tamanho pequeno como as mediterrâneas. A população caracterizou-se por apresentar indivíduos com diferentes graus de mestiçagem em consequência dos cruzamentos que lhes deram origem, o que justificou também sua maior variação fenotípica.

Alguns indivíduos da raça Peloco tiveram desempenho capaz de agrupá-los com as linhagens comerciais (Figura 1), o que mostra que esta variação fenotípica da raça Peloco abrange características de produção. A presença de variação fenotípica nas galinhas Peloco pode ser interpretada como fonte de diversidade genética e pode auxiliar a seleção em eventuais programas de melhoramento da raça. Em trabalhos futuros, podem também ser utilizadas em cruzamentos com o objetivo de complementariedade das características adaptativas (resistência a doenças e a estresse térmico), geralmente encontradas nas raças nativas, com as características de desempenho das linhagens comerciais.

A análise de agrupamento, realizada pelo método de otimização de Tocher, permitiu a formação de dois grupos. No grupo I, foram alocadas as linhagens comerciais L1, L2 e L3 e, no grupo II, manteve-se isolada a raça Peloco. O dendrograma gerado pelo método UPGMA corroborou o método de Tocher

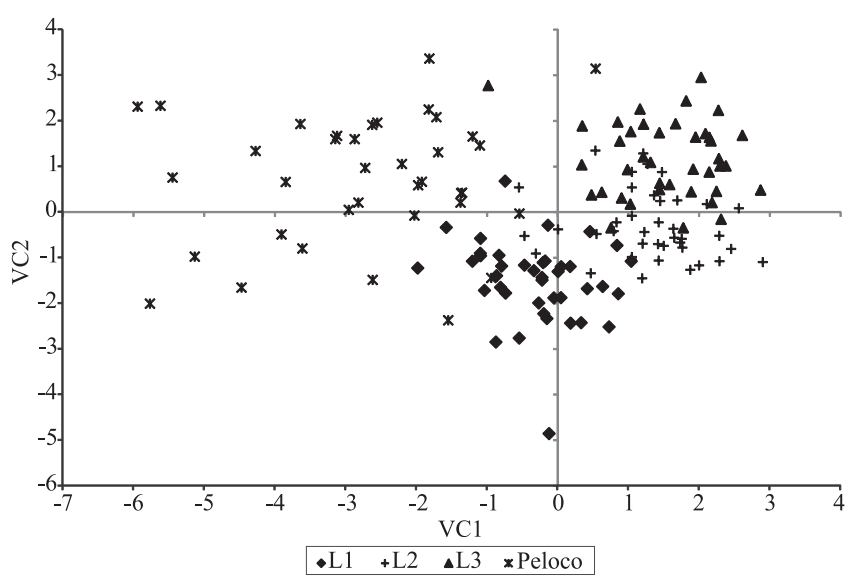

Figura 1. Dispersão gráfica da distância intrapopulacional das linhagens L1, L2 e L3 e da raça naturalizada Peloco, em relação às variáveis canônicas (VC1 e VC2) obtidas a partir das medidas de carcaça.

Pesq. agropec. bras., Brasília, v.48, n.11, p.1517-1523, nov. 2013 DOI: 10.1590/S0100-204X2013001100013 
no isolamento das aves Peloco, tendo-se obtido dois grupos com bootstrap de $100 \%$ (Figura 2). Ao se considerar o bootstrap de $65 \%$, observou-se a formação de três grupos: 1, raça Peloco; 2, linhagens L2 e L3; e 3, linhagem L1. Valores de bootstrap acima de 50\% indicaram maior confiança na formação dos nódulos do dendrograma.

A elevada variação do peso corporal entre a raça Peloco e as linhagens comerciais influenciou as características de carcaça, o que não permitiu o agrupamento dessas aves. No entanto, é necessária uma avaliação cautelosa a respeito do melhoramento das raças nativas. É necessário estabelecer-se um programa para melhorar as características produtivas, sem que haja perda da identidade da raça, fator que agrega valor ao produto final. Assim, obter animais maiores e mais pesados pode não ser o principal objetivo.

A Peloco é uma raça leve ao abate (com 150 dias de idade), e essa característica pode ser um diferencial destas aves. Além disso, nota-se a necessidade de melhorar a padronização das carcaças, de forma que o produtor consiga comercializá-las em um fluxo constante. Assim, é importante estabelecer como objetivo da seleção a uniformidade do crescimento, do ganho de peso e da conversão alimentar, bem como uma avaliação criteriosa dos índices reprodutivos das

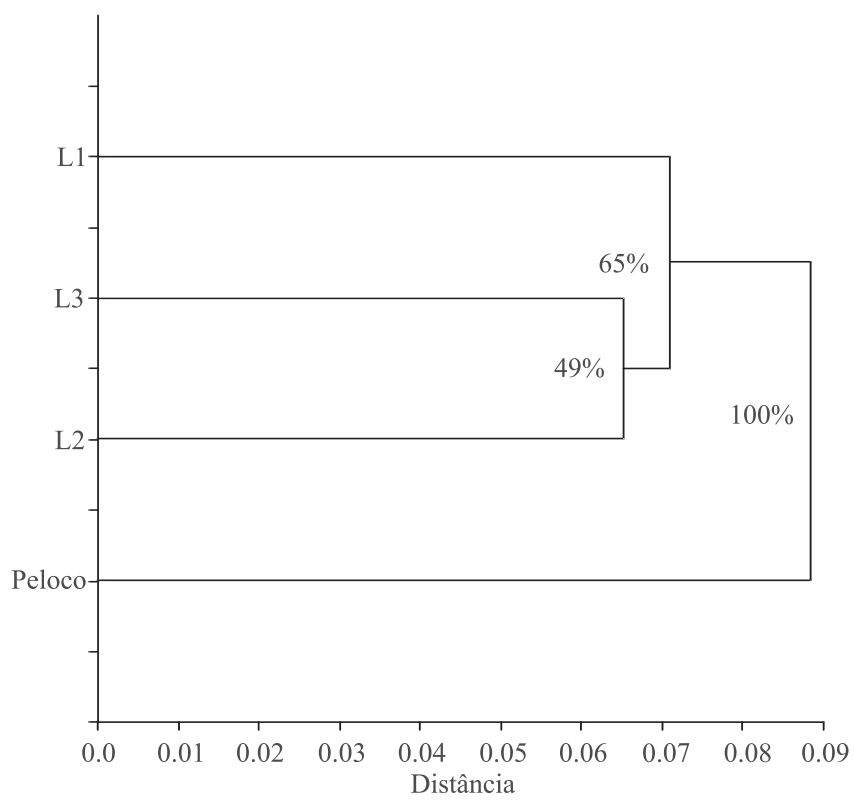

Figura 2. Dendrograma obtido a partir da distância de Mahalanobis $\left(\mathrm{D}^{2}\right)$, pelo método de agrupamento UPGMA, para as linhagens L1, L2 e L3 e a raça naturalizada Peloco. aves, como fertilidade, eclodibilidade e sobrevivência dos pintainhos, entre outras.

\section{Conclusões}

1. As galinhas nativas Peloco têm ampla variabilidade fenotípica quanto às características de carcaça, com grande divergência dos fenótipos comerciais.

2. As galinhas nativas Peloco têm menor peso de carcaça do que as de linhagens comerciais.

3. A grande variabilidade intrapopulacional apresentada pelas galinhas Peloco indica a possibilidade de seleção e a necessidade de padronização das carcaças e uniformidade de crescimento desta raça.

\section{Referências}

ADELEKE, M.A.; PETERS, S.O.; OZOJE, M.O.; IKEOBI, C.O.N.; BAMGBOSE, A.M.; ADEBAMBO, O.A. Growth performance of Nigerian local chickens in crosses involving an exotic broiler breeder. Tropical Animal Health and Production, v.43, p.643-650, 2011. DOI: 10.1007/s11250-010-9747-3.

AJAYI, O.O.; ADELEKE, M.A.; SANNI, M.T.; YAKUBU, A.; PETERS, S.O.; IMUMORIN, I.G.; OZOJE, M.O.; IKEOBI, C.O.N.; ADEBAMBO, O.A. Application of principal component and discriminant analyses to morpho-structural indices of indigenous and exotic chickens raised under intensive management system. Tropical Animal Health and Production, v.44, p.1247-1254, 2012. DOI: 10.1007/s11250-011-0065-1.

CRUZ, C.D. Programa Genes: diversidade genética. Viçosa: Ed. da UFV, 2009. 382p.

CRUZ, C.D.; CARNEIRO, P.C.S. Modelos biométricos aplicados ao melhoramento genético. 2.ed. Viçosa: Ed. da UFV, 2006. 585p.

CRUZ, C.D.; FERREIRA, F.M.; PESSONI, L.A. Biometria aplicada ao estudo da diversidade genética. Viçosa: Ed. da UFV, 2011. 620p.

EGITO, A.A.; MARIANTE, A.S.; ALBUQUERQUE, M.S.M. Programa brasileiro de conservação de recursos genéticos animais. Archivos de Zootecnia, v.51, p.39-52, 2002.

FRANCO, D.; ROIS, D.; VAZQUEZ, J.A.; LORENZO, J.M. Comparison of growth performance, carcass components, and meat quality between Mos rooster (Galician indigenous breed) and Sasso T-44 line slaughtered at 10 months. Poultry Science, v.91, p.1227-1239, 2012. DOI: 10.3382/ps.2011-01942.

HAILEMARIAM, M.; MULATU, D.; KELAY, B.; BERHAN, T. Assessment of the nutritional status of indigenous scavenging chickens in Ada'a district, Ethiopia. Tropical Animal Health and Production, v.42, p.123-130, 2010. DOI: 10.1007/ s11250-009-9395-7.

HAMMER, Ø.; HARPER, D.AT.; RYAN, P.D. PAST: paleontological statistic software package for education and data analysis. Paleontologia Electronica, v.4, p.1-9, 2001. 
KAYA, M.; YILDIZ, M.A. Genetic diversity among Turkish native chickens, Denizli and Gerze, estimated by microsatellite markers. Biochemical Genetics, v.46, p.480-491, 2008. DOI: 10.1007/ s10528-008-9164-8.

KGWATAlALA, P.M.; NOGAYAGE, M.; NSOSO, S.J. Growth performance of different strains of indigenous Tswana chickens under intensive management system. African Journal of Agricultural Research, v.7, p.2438-2445, 2012. DOI: 10.5897/ AJAR11.1220.

MAHALANOBIS, P.C. On the generalized distance in statistics. Proceedings of Natural Institute of Sciences, v.2, p.49-55, 1936.

OLIVEIRA, A.C.B. de; SEDIYAMA, M.A.N.; PEDROSA, M.W.; GARCIA, N.C.P.; GARCIA, S.L.R. Divergência genética e descarte de variáveis em alface cultivada sob sistema hidropônico. Acta Scientiarum. Agronomy, v.26, p.211-217, 2004. DOI: 10.4025/actasciagron.v26i2.1894.

PAIVA, S.R.; DERGAM, J.A.; MACHADO, F. Determining management units in Southeastern Brazil: the case of Astyanax bimaculatus (Linnaeus, 1758) (Teleostei: Ostariophysi: Characidae). Hydrobiologia, v.560, p.393-404, 2006. DOI: 10.1007/s10750-005-9415-1.

PERES-NETO, P.R. Introdução a análises morfométricas: tópicos em tratamento de dados biológicos. Oecologia Brasiliensis, v.3, p.57-89, 1995. DOI: 10.4257/oeco.1995.0201.03.
REIS, S.F.; PESSOA, L.M.; STRAUSS, R.E. Application of size-free canonical discriminant analysis to studies of geographic differentiation. Revista Brasileira de Genética, v.13, p.509-520, 1990.

ROSTAGNO, H.S. (Ed.). Tabelas brasileiras para aves e suínos: composição de alimentos e exigências nutricionais. 3.ed. Viçosa: Ed. da UFV, 2011. 252p.

SILVA, E.L. da; CENTOFANTE, L.; MIYAZAWA, C.S. Análise morfométrica em Thoracocharax stellatus (Kner, 1858) (Characiformes, Gasteropelecidae) proveniente de diferentes bacias hidrográficas sul-americanas. Biota Neotropical, v.9, p.71-76, 2009. DOI: 10.1590/S1676-06032009000200006.

SINGH, D. The relative importance of characters affecting genetic divergence. The Indian Journal of Genetics and Plant Breeding, v.41, p.237-245, 1981.

YAKUBU; UGBO, S.B. An assessment of biodiversity in morphological traits of Muscovy ducks in Nigeria using discriminant analysis. International Conference on Biology, Environment and Chemistry, v.1, p.389-391, 2011.

ZANETTI, E.; DE MARCHI, M.; DALVIT, C.; MOLETTE, C.; REMIGNON, H.; CASSANDRO, M. Carcase characteristics and qualitative meat traits of three Italian local chicken breeds. British Poultry Science, v.51, p.629-634, 2010. DOI: 10.1080/00071668.2010.521142.

Recebido em 8 de agosto de 2013 e aprovado em 30 de outubro de 2013 